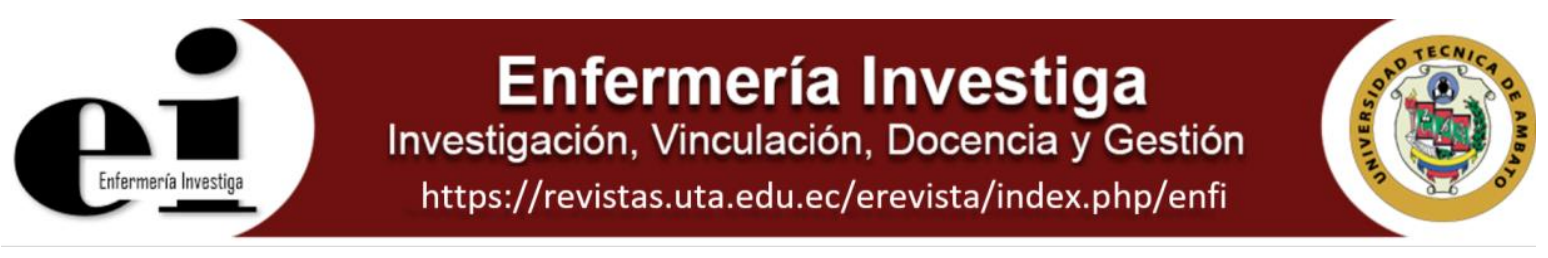

\title{
MALTRATO INFANTIL Y TRASTORNOS CLÍNICOS POST-VIOLENCIA EN NIÑOS MENORES DE CINCO AÑOS
}

\section{CHILD ABUSE AND POST-VIOLENCE CLINICAL DISORDERS IN CHILDREN UNDER FIVE YEARS}

María José Terán Bejarano ${ }^{1,2}$ https://orcid.org/0000-0002-3818-0284, Isabel Cluet de Rodriguez ${ }^{3}$ https://orcid.org/0000-0001-92285557, Vanessa Michelle Barzallo Puebla 4 https://orcid.org/0000-0003-0091-1309, Mónica Tatiana Escobar Suárez² https://orcid.org/0000-0003-1480-6075, Carlos Antonio Escobar Suárez 2,4https://orcid.org/0000-0002-9280-1555.

\author{
${ }^{1}$ Médico General del Hospital General Docente Ambato. Ambato, Ecuador. \\ 2Doctorante de Ciencias de la Salud, División de Estudios de Graduados. Facultad de Medicina Universidad de Zulia. Maracaibo, \\ Venezuela \\ ${ }^{3}$ Profesora Titular Emérito de la Escuela de Medicina, Departamento Pediátrico. Facultad de Medicina. Universidad del Zulia. \\ Maracaibo, Venezuela \\ ${ }^{4}$ Médico General en Funciones Hospitalarias, Área Auditoria Médica, Hospital Carlos Andrade Marín, Quito, Ecuador \\ ${ }^{5}$ Docente de la Universidad Central del Ecuador, Docente de la Universidad Autónoma de los Andes, Ecuador
}

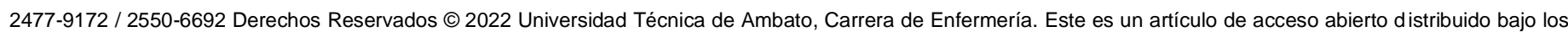

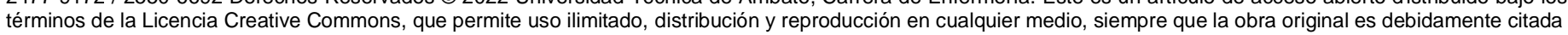

\section{RESUMEN}

Introducción: El maltrato infantil (MI), se ha convertido en un problema de salud pública que impregna todos los sectores sociales y cuyo impacto resulta devastador no solamente durante su infancia, sino a lo largo de toda su vida. Objetivo: Describir el maltrato infantil y trastornos clínicos post-violencia en niños menores de cinco años. Métodos: A documentary research was carried out in databases such as SciElo, Pubmed, Google Scholar and, LATINDEX, using the descriptors: child abuse, child abuse, family relationships, family violence, published in the period 2010-2021, in languages English, Spanish and Portuguese. Resultados: Se considera que, el maltrato infligido en los primeros cinco años de la vida del menor, repercute en el desarrollo neurobiológico y psicológico, el cual tiende a ser más rápido que en los años siguientes de las etapas del desarrollo. El maltrato infantil, no solo consiste en la presencia de hematomas, quemaduras, traumas craneales, la negligencia y la desnutrición. Se asume el maltrato infantil como todo aquel daño emocional que acompaña a los actos abusivos o negligentes que se traducen como trastorno de estrés agudo y el trastorno de estrés postraumático, con su repercusión a largo plazo. Conclusiones: El maltrato infantil puede afectar el exitoso desarrollo del niño no sólo en un determinado período de desarrollo, sino a lo largo de toda su vida. Un equipo multidisciplinario, que ejecute estrategias de prevención, la necesidad inclusión de todos los países en esta lucha y entender que la prevención es la única salida

Palabras claves: maltrato a los niños, abuso de los niños, relaciones familiares, violencia familiar

\section{ABSTRACT}

Introduction: Child abuse (MI) has become a public health problem that permeates all social sectors and whose impact is devastating not only during childhood, but throughout their entire lives. Objective: To describe child abuse and post-violence clinical disorders in children under five years of age. Methods: A documentary investigation was carried out. Through the search for scientific articles, in databases such as SciElo, Pubmed, Google Scholar and, LATINDEX, using the descriptors: child abuse, child abuse, family relationships, family violence. With inclusion criteria: full articles, in national and international journals and organizations such as WHO, PAHO, published in the period 2010-2021, in English, Spanish and Portuguese languages. Results: It is considered that the abuse inflicted in the first five years of the child's life affects the neurobiological and psychological development, which tends to be faster than in the following years of the development stages. Child abuse not only consists of bruises, burns, head trauma, neglect and malnutrition. Child abuse is assumed as all the emotional damage that accompanies abusive or negligent acts that translate as acute stress disorder and post-traumatic stress disorder, with its longterm repercussions. Conclusions: Child abuse can affect the successful development of the child not only in a certain period of development, but throughout their entire life. A multidisciplinary team, which executes prevention strategies, the need to include all countries in this fight, and understands that prevention is the only way out

Keywords: child abuse, child abuse, family relationships, family violence 


\section{INTRODUCCIÓN}

En el panorama mundial la violencia contra los niños es una preocupación global que encierra diferentes formas de manifestación que pueden repercutir en el trascurso de la vida y ser considerado un problema social por las diferentes expresiones sociales (1), resulta imperioso el análisis documental existente desde las primeras observaciones del fenómeno hasta las actuales y darle un seguimiento histórico para reconocer y entender el nuevo paradigma clínico, aspectos que hasta ahora apenas se comprenden(2). En tal sentido, todas la organizaciones inherentes en el cuidado y búsqueda de la equidad social deben buscar formas y métodos que ayuden a disminuir a las víctimas por este fenómeno que de por si es preocupante.

En este contexto se puede citar investigaciones de la Organización Mundial de Salud (OMS) donde se demuestra cifras mundiales reales de casi 265 millones de niños maltratados en una o varias formas. Por tanto, se relaciona a este hecho como causa de alteraciones en la salud mental y física que perduran toda la vida, el nivel socio-profesional puede enlentecer el desarrollo económico y social de los puntos focales donde se encuentra la violencia con los niños; cada país desde su perspectiva enfrenta este hecho tratando de articular cada variable para su mejor tratamiento, como el manejo de estrés postraumático, secuelas sicológicas innegables como depresión , ansiedad y el intento autolítico (3).

A nivel mundial, el $3 \%$ de niños de 2 a 4 años, sufren algún tipo de violencia por parte de la persona cuidadora. Se estima que 250 millones de jóvenes alrededor del mundo, es decir 6 de cada 10 niños entre 2 y 14 años de edad sufren de manera periódica castigos físicos a manos de sus cuidadores. En treinta países que se realizó un estudio demuestra que seis de cada diez niños entre 12 a 23 meses están sometidos a algún tipo de disciplina violenta (4). Entre los niños de esta edad, casi la mitad son víctimas de castigos físicos y una proporción similar están expuestos al abuso verbal. En la mayoría de los casos, los niños son objeto también de agresiones psicológicas (5).

En Latinoamérica y el Caribe (LAC) se han incrementado el número de estudios sobre la violencia sexual, al ser una problemática en estas dos últimas décadas en el perfil epidemiológico de la región. Casi la mitad de los estudios identificados provienen de Brasil y México, además se han realizado algunas investigaciones en Bolivia, Colombia, Guatemala, Haití, Jamaica, Nicaragua, Perú y la República Dominicana; pero sigue habiendo pocas referencias académicas dadas las condiciones peculiares que existe tanto el cultura, educación y políticas de manejo de datos(6).

Así también en México, la incidencia de maltrato infantil predomina en menores de tres años, ubicándose en el sexto lugar a nivel mundial. Colombia, para el año 1994 presentó 1861 niñas y 1352 niños víctimas de violencia, donde predominó agresiones físicas, psicológicas y finalmente sexuales (7). Del total de abusos sexuales a niños y adolescentes el 15\% realizó denuncia del caso y solo $5.3 \%$ recibió una sanción o penalización jurídica. Actualmente, la violencia contra niños se ha incrementado por muchas razones, como la cultura de violencia familiar, falta de desarrollo económico e inequidad social por parte de los gobiernos de turnos, con panoramas poco alentadores (8).
Un caso que requiere de atención es la realidad de Ecuador, país que registró en el año 2010 maltrato infantil en cuatro de cada diez niños, niñas y adolescentes; entre el grupo etario de cinco a diecisiete años recibieron un trato violento de sus padres por algún desacuerdo familiar(9). En 2017, en sala de primera acogida del Hospital General Docente Ambato, una de las salas en el país con mayor experiencia en el campo de la atención a víctimas de cualquier tipo de violencia se identificaron un total de 202 pacientes que han acudido espontáneamente, de los cuales 94 fueron niñas y 108 niños, con evidencias de maltrato físico, agresión sexual y/o casos de negligencia por parte de los cuidadores designados (10).

Las implicaciones del maltrato infantil para los servicios de salud e instituciones de justicia, frente a una creciente necesidad de atención médica por las víctimas de este tipo, la han constituido como una enfermedad de dimensiones impensables en este siglo (11). El objetivo del presente estudio es describir el maltrato infantil y trastornos clínicos post violencia en niños menores de cinco años

\section{MÉTODOS}

Se realizó una investigación de diseño documental, de carácter descriptivo y exploratorio. A través de la búsqueda de artículos científicos completos, en bases de datos como SciElo, Pubmed, Google Scholar and LATINDEX, utilizando los descriptores: maltrato a los niños, abuso de los niños, relaciones familiares, violencia familiar.

Dentro de los criterios de inclusión se utilizó, artículos completos, de revistas nacionales e internacionales y organizaciones como OMS, OPS, publicados en el periodo 2010-2021, en los idiomas inglés, español y portugués. Se excluyeron información no relevante, artículos repetidos, versiones incompletas y que no cumplan con los criterios de inclusión.

Se cumplieron normas y principios universales de ética establecidos a fin, de evitar la difusión de información con fines deshonestos y garantizar la total transparencia en la investigación, así como se resguardo la propiedad intelectual de los autores.

\section{RESULTADOS Y DISCUSIÓN}

Se identificaron inicialmente noventa y cinco artículos escritos, en idioma inglés, español y portugués, sobre los aspectos a investigar, de los cuales una vez clasificados y revisados sus resúmenes se seleccionaron treinta y dos artículos que cumplían con los criterios de inclusión ya señalados.

Hoy en día las investigaciones en el área del maltrato infantil aún con ciertas lagunas conceptuales- realizan diversos abordajes en aspectos como: epidemiología, definición, consecuencias del desarrollo, resultados a largo plazo e intervenciones; lo que ha permitido comprender el cómo el maltrato infantil ejerce un impacto devastador y prolongado en los niños y su incidencia en el costo social (12).

Al-Ali et al., en su investigación expresan que los niños abusados y objetos de negligencia, eran 1.8 veces más proclives a ser arrestados como delincuentes juveniles que aquellos que no 
habían sufrido(13). Los niños maltratados son en suma más propensos a desarrollar problemas de abuso de sustancias psicotrópicas, indicando que más del $50 \%$ de los niños maltratados tiene dificultades en la escuela requiriendo en ello, servicios especiales de educación(14).

Entender el maltrato infantil implica un desafío, en los últimos años se ha incrementado el número de niños que ha experimentado alguna forma de maltrato. Un estudio longitudinal, con duración de 7 años, realizado en los Estados Unidos en 1993 expresa que casi 1.6 millones de niños norteamericanos fueron abusados o tratados con negligencia (15). Observándose que 111 de cada mil, fueron objeto de maltrato y trece de negligencia. Entre esos niños maltratados, $50 \%$ tenían menos de siete años $(15,16)$.

Estudios revelan con crudeza que el maltrato infantil supera cualquier concepto tradicional antes concebido e irrumpe con un nuevo paradigma teórico en las ciencias médicas, por un lado alerta sobre un flagelo que crece exponencialmente en todos los estratos sociales, a la vez que permite abordar todas las aristas del maltrato, lo que permite ahora establecer un concepto integrador y totalizador entendiéndolo como: "toda conducta de acción u omisión que provoque o pueda provocar daño a la integridad o salud física, psicológica o sexual a los niños por parte de cualquier persona incluidos sus progenitores, otros parientes, educadores, e inclusive personas a cargo de su cuidado; cualquiera que sea el medio utilizado para el efecto, observándose para ello sus consecuencias , así como el tiempo necesario para la recuperación de la víctima"(17). Se incluye en esta calificación: "el trato sobre la negligencia o descuido grave o reiterado en el cumplimiento de las obligaciones para con los niños, niñas y adolescentes, relativas a la prestación de alimentos, atención médica, educación, cuidados diarios y su utilización en la mendicidad"(12).

Desde esta perspectiva, entender el maltrato infantil resulta devastador para cualquiera que se enfrente a la tipificación de un delito de complejo aspecto, pues que no sólo afecta la "psique", sino el alma, el cuerpo y la esfera emocional de quien lo transita dejando en su genética una impronta improbable de superación o de sanación integral (18). Por ello la necesidad de un abordaje de amplio espectro; no obstante, pareciera ser éste un tema que continúa enfrentando desafíos singulares que abarcan temas álgidos tales como: -la falta de un claro criterio operacional frente a los actos que constituyen maltrato infantil (19); -la controversia con respecto a la mejor forma de verificar la ocurrencia del maltrato, -la repercusión en resultados sesgados al obtener como conclusiones, que rara vez experimentan un sólo tipo de abuso, -la falsa creencia ante el maltrato como conducta más habitual en familias de bajos ingresos, -el poco o exiguo avance en explicar los procesos y mecanismos que contribuyen a la serie de consecuencias del desarrollo observados en niños maltratados, -el enfoque unilateral observados en los resultados conductuales y psicológicos sobre el maltrato infantil durante los cinco primeros años sin considerar las secuelas neurobiológicas y la negligencia (20).

Ante todo, lo antes expuesto, se suma irremediablemente la heterogeneidad de resultados entre los niños maltratados, lo que sugiere que el maltrato no afecta a todos los pequeños en forma similar. La variedad de resultados acentúan la importancia de examinar a los predictores de la resiliencia, a pesar de la adversidad del maltrato; y finalmente quizás el mayor desafío estriba en la prevención, pues se está en presencia de la existencia de estrategias efectivas de prevención e intervención del maltrato infantil, lo que es en definitiva el mecanismo más idóneo para exterminar este azote social (21).

\section{El maltrato infantil y sus perspectivas como disciplina}

Las investigaciones sobre las consecuencias socioemocionales del maltrato durante los primeros años de vida, proporcionan ahora una base de conocimientos respecto a la forma adversa o negativa que puede erosionar el desarrollo. No obstante, las disciplinas convergentes en la materia deben intentar por su parte formular y evaluar cada caso bajo la premisa de la prevención y ejecución de estrategias para intervención de los niños y familias vulnerables (22). No cabe duda que quizá, una de las acciones más inmediatas es definir científicamente este tema como un área clínica y subclínica, que permita intervenir lo más rápidamente posible después de identificado el maltrato incluso si no se manifiestan de inmediato síntomas de una enfermedad mental diagnosticable- para referir al paciente a un especialista en salud mental, que estudie la posible necesidad de una intervención temprana, a fin de mitigar consecuencias negativas de desarrollo (23).

Otro aspecto importante, es el abuso materno de sustancias psicotrópicas y la subsecuente incapacidad para cuidar de los niños, que constituye una de las razones de peso bajo al nacer y durante la infancia, cada vez más frecuente. Por lo tanto es necesario investigar los efectos del maltrato y la exposición pre y post natales a las sustancias psicotrópicas y sus implicaciones (24).

Es parte del quehacer científico de esta disciplina, poner en marcha capacitaciones para quienes luchan con aspectos relativos al maltrato infantil y la negligencia. Es un deber para quienes trabajan en la protección de los niños, buscar información relacionada con la importancia de proporcionar intervenciones psicológicas a los niños pequeños que han sido maltratados y a sus cuidadores biológicos o adoptivos (25). El personal de servicio a cargo de la protección debe mantener la actualización de las investigaciones respecto a los efectos de corto y largo plazo del maltrato, incrementar sus esfuerzos enviando a estos niños a evaluación psicológicas antes que aparezcan desórdenes conductuales o psiquiátricos.

Estos programas deben manejar conocimientos básicos sobre el desarrollo infantil, puesto que las consecuencias del maltrato pueden variar según el período de desarrollo durante el cual se produce el trauma, y para ello los terapeutas deben estar preparados. En síntesis, para encarar el maltrato en la niñez y adolescencia, es necesario realizar esfuerzos para superar brechas apelando todos los recursos disponibles, aplicar correctivos, así como en las políticas estatales de cada país o nación(26).

Como se ha podido observar, el maltrato infantil, requiere de servicios y cuidados extremos posteriores; siendo necesario, dar seguimiento a los menores de cinco años de edad víctimas de maltrato, dando respuestas adecuadas que ayuden al niño en su desarrollo evolutivo, construyendo estrategias que eviten agresiones futuras, en aquellos grupos vulnerable $y$ susceptibles, que en muchas ocasiones ni siquiera pueden expresarse adecuadamente o simplemente son incapaces de hacerlo (27). La identificación de $\mathrm{Ml}$, es un paso previo e 
imprescindible para la actuación y orientación de la problemática, para encaminar medidas preventivas (17).

Además de conocer los trastornos clínicos y conductuales post violencia, es necesario investigar los factores de riesgo asociados y las necesidades de prevención y atención a este grupo vulnerable, para reducir a corto plazo el reincidente riesgo de maltrato, interrumpiendo el ciclo de la violencia, minimizando sus consecuencias clínicas y psicológicas postraumáticas al evento de violencia (1). Dentro de los factores de riesgos para que se desarrolle o no un trastorno de estrés post traumático se encuentra: La gravedad del acontecimiento traumático, si se produjeron lesiones físicas durante el acontecimiento, el carácter del niño, I estatus social y económico de la familia, si el niño ha experimentado anteriormente la adversidad (como el abuso sexual), el buen funcionamiento de la familia, si el niño tiene familiares con trastornos de salud mental y si el niño tiene apoyo familiar y social (1).

\section{Trastornos clínicos post violencia}

Dentro de los trastornos clínicos y conductuales, podemos observar: los trastornos de estrés agudo y postraumático, que son reacciones a hechos traumáticos abrumadores que incluyen recuerdos repetitivos e intrusivos sobre el hecho, así como embotamiento emocional y aumento de la tensión o el estado de alerta. Los niños tienden a evitar aquellas situaciones que les recuerden el evento. El trastorno se desarrolla después de que el niño haya presenciado o experimentado un acto de violencia. El niño no solo revive el suceso, sino que también puede sentirse paralizado a nivel emocional, extremadamente tenso y nervioso. El diagnóstico se basa en los síntomas que aparecen después de un acontecimiento traumático $(28,29,30)$

El trastorno por estrés agudo, suele empezar inmediatamente después del acontecimiento traumático y dura entre 3 días y 1 mes. El trastorno de estrés postraumático puede ser una continuación del trastorno por estrés agudo o no desarrollarse hasta 6 meses después del evento. Los trastornos de estrés pueden aparecer después de que el niño presencie 0 experimente un suceso. Durante el suceso, habitualmente siente miedo intenso, desamparo $u$ horror. Estos sucesos incluyen actos de violencia como el maltrato infantil. En los niños pequeños, la violencia doméstica es la causa más frecuente. No todos los niños que experimentan un suceso traumático desarrollan el trastorno. Los niños no tienen que experimentar directamente el evento traumático. Pueden desarrollar un trastorno de estrés si son testigos de un evento traumático que ocurre a otros $(28,29,30)$

Los síntomas del trastorno de estrés agudo y el trastorno de estrés postraumático son similares e incluyen varios tipos de síntomas. Dentro de los cuales se encuentra: Reexperimentación del acontecimiento traumático (síntomas de intrusión). El niño puede revivir el acontecimiento traumático mientras está despierto (escena retrospectiva o flash-back) o en sueños (pesadillas). Las escenas retrospectivas (flash-back) suelen desencadenarse por algo asociado con el suceso original. Durante la escena retrospectiva, el niño se muestra aterrorizado y desconectado del entorno. Puede perder temporalmente el contacto con la realidad e intenta frenéticamente esconderse o huir, actuando como si estuviera en peligro. De forma menos radical, el niño revive a veces el suceso en forma de pensamientos, imágenes mentales 0 recuerdos que son, sin embargo, muy angustiosos.
Frecuentemente, los niños pequeños pueden recrear el acontecimiento durante el juego. -Evitar recordatorios (síntomas de evitación). Los niños pueden evitar constantemente todo aquello que les recuerde el trauma, ya sean actividades, situaciones 0 personas. Incluso pueden intentar evitar pensamientos, sentimientos o conversaciones sobre el suceso traumático. Normalmente fracasa en sus intentos de dejar de recordar el suceso. -Efectos negativos sobre el pensamiento y el estado de ánimo. Es frecuente sentirse paralizado emocionalmente o separado del propio cuerpo. El niño afectado pierde el interés en sus actividades normales, se aísla de otras personas y se preocupa por la muerte a una edad temprana. El niño se siente culpable, por ejemplo, por no haber podido hacer nada para impedir o detener el suceso. No pueden recordar detalles importantes del evento o los pueden recordar de manera incorrecta. Puede, por ejemplo, pensar que fue su culpa.Cambios en las reacciones y el estado de alerta. Los niños afectados pueden mostrarse excesivamente pendientes de cualquier señal de peligro. Se siente extremadamente tenso (hiperactivación), es incapaz de relajarse y se sobresalta fácilmente. Les cuesta controlar sus reacciones, lo que da lugar a una conducta imprudente o a arranques de ira. Pueden tener dificultad para relajarse, para dormir o para concentrarse. Síntomas disociativos. Los niños se sienten separados de su cuerpo como en un sueño. También sienten que el mundo es irreal $(29,30)$. El tratamiento consiste en psicoterapia, terapia conductual y fármacos.

La importancia del tamizaje y el abordaje de cualquier tipo de agresión, según marca el protocolo reflejado en la norma técnica de atención en violencia, facilita la proximidad y la continuidad de la atención sanitaria, lo que se traduce en políticas públicas de estado integrales para la atención y seguridad al menor; dado que el MI, ha sido y será un problema de política pública de impacto social con incidencia directa en los índices de morbimortalidad a nivel mundial y de desarrollo de cada nación(31). Los gobiernos necesitan aceptar que es una emergencia real. Ya que durante siglos los niños y niñas han sufrido violencia a manos de los adultos sin que fuera vista ni oída. Pero ahora que la escala e impacto de la violencia contra los niños y niñas se ha hecho visible, se debe ejecutar una protección efectiva a la cual tienen derecho de manera incondicional (32).

La implementación y cumplimiento de protocolos de acceso inmediato y prioritario para la atención especializada e integral, son las rutas de acceso a la justicia de los organismos locales, para poder obtener de forma rápida las medidas de protección y garantizar en definitiva el bien- estar del menor de edad (33). En síntesis, estas políticas públicas orientadas a la prevención y reinserción del niño maltratado, son recursos suficientes para erradicar este delito tipificado como el mal de las sociedades en la actualidad (34-36).

\section{CONCLUSIONES}

El maltrato infantil puede afectar el exitoso desarrollo del niño no sólo en un determinado período de desarrollo, sino a lo largo de toda su vida. Es importante reconocer. Se han aclarado nuevos e importantes aspectos de investigación en torno a este crítico problema de toda la sociedad. Existe la necesidad imperiosa de más investigaciones respecto a los efectos del maltrato infantil durante la infancia y la niñez, así como el desarrollo e implementación de investigaciones amplias y longitudinales respecto al maltrato infantil y sobre los factores de riesgo coexistentes. La necesidad de un equipo multidisciplinario, que 
ejecuten estrategias de prevención de este delito, la necesidad inclusión de todos los países en esta lucha, y entender que la prevención es la única salida para proteger a los niños y niñas contra la violencia, con el fin de reducir todas las formas de violencia en la sociedad, así como las consecuencias sociales y de salud integral.

\section{REFERENCIAS}

1. World Health Organization. Responding to children and adolescents who have been sexually abused: WHO clinical guidelines. World Health Organization. 2017.Disponible en: https://apps.who.int/iris/handle/10665/259270.

2. Paulo Sérgio Pinheiro. Informe mundial sobre la violencia contra los niños y niñas. Estudio del Secretario General de las Naciones Unidas sobre la Violencia contra los Niños. 2006, Disponible en: https://www.unicef.org/mexico/spanish/Informe Mundial Sobre Violencia.pdf.

3. World Health Organization. Global status report on violence prevention 2014. 2014. Disponible en: https://www.who.int/publications//item/9789241564793

4. Cecilie Modovar, María Elena Ubeda. Fondo de las Naciones Unidas para la Infancia (UNICEF). La violencia en la primera infancia. Marco Regional de UNICEF para América Latina y el Caribe Secciones de Desarrollo de la Primera Infancia y Protección Infantil. Panamá, 2017. ISBN: $\quad 978-92-806-4908-6 . \quad$ Disponible en: https://www.unicef.org/lac/media/686/file/PDF\%20La\%20violencia\%20en\%20la\%20primera\%20infancia

5. Lenzer Gertrud. Violence against children. Routledge International Handbook of Children's Rights Studies. Routledge, 2015:292310.

https://scholar.google.com.ec/scholar?q=Lenzer+G.+Violence+against+children\&hl=es\&as_sdt=0\&as_vis=1\&oi=scholart.

6. Devries K, Merrill KG, Knight L, Bott S, Guedes A, Butron-Riveros B, et al. Violence against children in Latin America and the Caribbean: What do available data reveal about prevalence and perpetrators? Rev Panam Salud Publica/Pan Am J Public Heal. 2019;43:1-11.

7. Brathwaite B. An Exploration of Youth Risks in the Caribbean, through the voices of youth. 2009;16. Disponible en: https://sta.uwi.edu/conferences/09/salises/documents/B Brathwaithe.pdf.

8. Tovar Domínguez, Aida Gisell, Almeraya Quintero, Silvia Xochilt, Guajardo Hernández, Lenin Gerardo, \& Borja Bravo, Mercedes. El maltrato infantil desde la voz de la niñez. Revista mexicana de ciencias agrícolas, 2016;7(1):195-207. Disponible en:http://www.scielo.org.mx/scielo.php?script=sci_arttext\&pid=S2007-09342016000100195\&lng=es\&tlng=es.

9. Comisión Económica para América Latina y el Caribe (CEPAL) y la Oficina Regional para América Latina y el Caribe del Fondo de las Naciones Unidas para la Infancia (UNICEF). Violencia contra niñas, niños y adolescentes en tiempos de COVID-19. 2020. Disponible en: https://www.unicef.org/lac/media/19611/file/violencia-contra-nna-en-tiempos-de-covid19.pdf

10. Mendoza Ureta RE, Reiban Garnica DI, Normativo M. El Abuso Sexual en instituciones educativas del Ecuador. 2013;2(2):5. Disponible en: http://revistas.unae.edu.ec/index.php/illari/article/download/268/224/

11. Osofsky joy D. The Im pa ct of Vi o I e n c e o n C h il dre n. 1999;9:30-45.

12. Pereira R, Loinaz I, del Hoyo-Bilbao J, Arrospide J, Bertino L, Calvo A, et al. Proposal for a definition of filio-parental violence: Consensus of the Spanish society for the study of filio-parental violence (SEVIFIP). Papeles del Psicol. 2017;38(3):216-23. Disponible en: http://www.psychologistpapers.com/English/2839.pdf

13. Al-Ali NM, Yaghy HS, Shattnawi KK, Al-Shdayfat NM. Parents' Knowledge and Beliefs about the Impact of Exposure to Media Violence on Children's Aggression. Issues Ment Health Nurs. 2018;39(7):592-529. Disponible en: https://doi.org/10.1080/01612840.2017.1422201

14. de Robertis C. La relación de ayuda en trabajo social. Fundam del Trab Soc Ética y Metodol. 2003;

15. School violence and bullying: Global status and trends, drivers and consequences, UNESCO. and Bullying: 2018. Disponible en: https://www.observatoriodelainfancia.es/oia/esp/documentos_ficha.aspx?id=5805

16. Fulu, E., Warner, X., Miedema, S., Jewkes, R., Roselli, T. and Lang, J. (2013). Why Do Some Men Use Violence Against Women and How Can We Prevent It? Quantitative Findings from the United Nations Multi-country Study on Men and Violence in Asia and the Pacific. Bangkok: UNDP, UNFPA, UN Women and UNV, 2013: 121. Disponible en: https://www.undp.org/content/dam/rbap/docs/Research \& Publications/womens_empowerment/RBAP-Gender-2013-P4P-VAWReport-Summary.pdf

17. National Institute of Mental Health NIH. Cómo ayudar a los niños y adolescentes a superar la violencia y otras experiencias traumáticas. Lo que los padres de familia, los socorristas y la comunidad pueden hacer. Disponible en: https://www.nimh.nih.gov/sites/default/files/documents/health/publications/espanol/como-ayudar-los-ninos/19-mh8066s_helpingchildrencopewithdisaster_spanish.pdf

18. Kann, Laura, Tim McManus, William A. Harris, Shari L. Shanklin, Katherine H. Flint, Joseph Hawkins, Barbara Queen, et al. Youth Risk Behavior Surveillance - United States, 2015. Morbidity and Mortality Weekly Report: Surveillance Summaries, 2016; 65(6):1-174. Disponible en: https://www.jstor.org/stable/24805802.

19. Santamaría Galeano CL, Tapia Varas PA. Violencia contra niños y adolescentes ejercida por cuidadores. Inf psicológicos. 2018;18(1):13-34. http://dx.doi.org/10.18566/infpsic.v18n1a01

20. Save the Children. End Violence Against Children Now End Violence. 2016;1-6. Available from: www.raddabarnen.se

21. McKenzie B, Kufeldt K. Canadian incidence study of reported child abuse and neglect: Themes and implications. Child Welfare: Connecting Research, Policy, and Practice: Second Edition. 2011:89-97.

22. Irene V. Intebi. Estrategias y modalidades de intervencion en abuso sexual infantil intrafamiliar. 2012. Disponible en: https://www.serviciossocialescantabria.org/uploads/documentos\%20e\%20informes/Estrategias\%20y\%20modalidades\%20de\%20in tervencion\%20en\%20abuso\%20sexual\%20infantil\%20intrafamiliar\%20\%20Marzo\%202012.pdf

23. Fernández Gerardo, Arráiz Sánchez Carolina, Gonzalo Paredes Rivas. Estrategias de prevención de violencia familiar de la cátedra 
escuela para padres: (Premio: fundación patronato hospital de niños J.M. de los Ríos. LIII congreso nacional de pediatría 2007). Arch Venez Puer Ped. 2008; 71(4):124-130. Disponible en: http://ve.scielo.org/scielo.php?script=sci_arttext\&pid=S000406492008000400005\&lng=es.

24. Gershoff ET. School corporal punishment in global perspective: prevalence, outcomes, and efforts at intervention. Psychol Heal Med. 2017;22:224-239. Available from: http://dx.doi.org/10.1080/13548506.2016.1271955

25. Pereda Beltrán N. ¿Uno de cada cinco? Victimización sexual infantil en España. Papeles del Psicol. 2016;37(2):126-33. Disponible en: http://www.papelesdelpsicologo.es/pdf/2697.pdf

26. World Health Organization. Global Plan of Action. 2007;482. Disponible en: https://www.who.int/reproductivehealth/publications/violence/global-plan-of-action/en/

27. González R, Martínez A, Rdenas A, Fernández A, Fernández A, Montero L, et al. Violencia doméstica en niños y adolescentes de la Comuna Tala Hady. Arch méd Camaguey. 2019;23(2):178-87. Disponible en: http://scielo.sld.cu/scielo.php?script=sci_arttext\&pid=S1025-

02552019000200178\&lang=es\&fbclid=IwAR22IXXBhhNrmywemkP6nN_QRkDBWloiuwNsGCtMIDYxM9kjGptToXluVt0

28. Martínez Almodóvar Melvis Beatriz, López Triana Adelaida E, Díaz Montesinos Arián, Teseiro Plá Modesta Mercedes. Violencia intrafamiliar y trastornos psicológicos en niños y adolescentes del área de salud de Versalles, Matanzas. Rev.Med.Electrón. 2015 ; 37(3):237-245. Disponible en: http://scielo.sld.cu/scielo.php?script=sci_arttext\&pid=S1684-18242015000300006\&lng=es.

29. Fátima Valencia Agudo. Maltrato en la infancia y la adolescencia: características y psicopatología asociada en un Centro de Salud Mental. Psicopatol. salud ment. 2016; 27: 49-58. Disponible en: https://www.fundacioorienta.com/wpcontent/uploads/2019/02/Valencia-F-R27.pdf

30. Rincón G Paulina, Cova S Félix, Bustos T Patricia, Aedo S Jaime, Valdivia P Mario. Estrés Postraumático en Niños y Adolescentes Abusados Sexualmente. Rev. chil. pediatr. 2010; 81(3): 234-240. Disponible en: http://www.scielo.cl/scielo.php?script=sci_arttext\&pid=S0370-41062010000300006\&lng=es. http://dx.doi.org/10.4067/S037041062010000300006

31. Sabino FHO, Aragão ADS, Sousa RM de, Querino RA, Santos HH Dos, Ferriani M das GC. A rede de proteção a crianças e adolescentes vítimas de violências: antigos dilemas, novas perspectivas. Rev Família, Ciclos Vida e Saúde no Context Soc. 2020;2(8):736. Disponible en: https://www.redalyc.org/articulo.oa?id=497965721009

32. Organización Panamericana de la salud. OPS. Violencia en las Américas ¿Qué debe hacerse? 2019;137(3):2015407. https://www.paho.org/sites/default/files/2020-11/WEB-PAHO_violence_triptico_ES.pdf

33. Fernández, G., De Fernández, C. A., Troya, E., Raza, C., Valencia, P., Fernández, M., \& López, J. La violencia doméstica: una experiencia adversa significativa con grandes complicaciones en el niño. Archivos Venezolanos de Farmacología y Terapéutica. 2019; 38(3): 140-147. Disponible en: https://www.proquest.com/openview/6f907f24a51017219d8217884deaba7b/1?pqorigsite $=$ gscholar $\& \mathrm{cbl}=1216408$

34. Hillis S, Mercy J, Amobi A, Kress H. Global prevalence of past-year violence against children: A systematic review and minimum estimates. Pediatrics. 2016;137(3). https://doi.org/10.1542/peds.2015-4079

35. Fernández, G., Farina, P., de Fernández, C. A., \& Ortiz, E. V. T. Consecuencias del maltrato infantil en un hospital de Maracaibo-

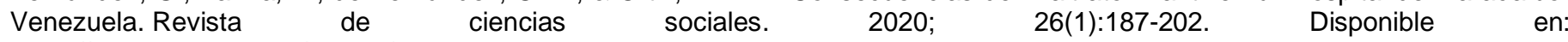
https://dialnet.unirioja.es/servlet/articulo?codigo $=7384414$

36. Ernst, Miriam. Los delitos sexuales en el Ecuador. Un análisis desde la experiencia. En: Aportes Andinos Quito: Universidad Andina Simón Bolívar, Sede Ecuador; Programa Andino de Derechos Humanos, 2007; 18:10. Disponible en: https://repositorio.uasb.edu.ec/handle/10644/867 